\title{
On proximity relations for valuations dominating a two- dimensional regular local ring
}

José J. Aparicio, Angel Granja and Tomás Sánchez-Giralda

\begin{abstract}
The purpose of this paper is to define a new numerical invariant of valuations centered in a regular two-dimensional regular local ring. For this, we define a sequence of non-negative rational numbers $\delta_{\nu}=\left\{\delta_{\nu}(j)\right\}_{j \geq 0}$ which is determined by the proximity relations of the successive quadratic transformations at the points determined by a valuation $\nu$. This sequence is characterized by seven combinatorial properties, so that any sequence of non-negative rational numbers having the above properties is the sequence associated to a valuation.
\end{abstract}

\section{Introduction.}

Valuations centered in a two-dimensional regular local ring have been studied and classified by Zariski, Abhyankar and Lipman (see for example [1]). More recently, there has been a revival of interest in this subject (see [15], [13], [7], [9], ..).

The main purpose of this paper is to define a new numerical invariant of valuations centered in a regular two-dimensional local ring. One advantage of our invariant over those of [15] is that it works for a general regular local ring of dimension two; in particular, we do not assume that the residue field is algebraically closed. 
The idea of proximity to classify singularities of analytically irreducible plane curves was developed by Enriques (see [6]) and can be adapted to the situation above (see [7], [9], [13],...).

Several invariants can be associated to proximity relations (the refined proximity matrix, the multiplicity sequence, the semigroup-length sequence, ..., see [13]). Here we will introduce a new one which is a sequence of non-negative rational numbers $\delta_{\nu}=\left\{\delta_{\nu}(j)\right\}_{j \geq 0}$ (later called proximity sequence), where the proximity relations are codified.

In what follows, all rings considered will be commutative and with a unit element. For a local ring $R$, we will denote by $M(R)$ its maximal ideal.

Throughout this paper, $R$ will be a two-dimensional regular noetherian local ring and we will consider a fixed sequence

$$
R=R_{0} \subset R_{1} \subset \cdots \subset R_{n} \subset \cdots,
$$

where $R_{i+1}$ is a quadratic transform of $R_{i}$ (i.e. $R_{i+1}$ is a localization at a maximal ideal of a ring $R\left[x^{-1} M\left(R_{i}\right)\right]$ with $x \in M\left(R_{i}\right)$ and $x \notin$ $\left(M\left(R_{i}\right)\right)^{2}$.

For $i>0$, we will denote by

$$
e_{i-1}=\left[\frac{R_{i}}{M\left(R_{i}\right)}: \frac{R_{i-1}}{M\left(R_{i-1}\right)}\right] .
$$

It should be remembered that $S=\cup_{i \geq 0} R_{i}$ is a valuation ring. (See [1]). If $\nu$ is the valuation of $S$ then $\nu$ is the only valuation of the quotient field of $R$ centered at the maximal ideal of $R_{i}$ for all $i \geq 0$.

The main goal of the paper is the characterization of the properties of the proximity sequence in the following sense: the properties that a sequence of non-negative rational numbers $\{\delta(j)\}_{j>0}$ must satisfy in order to be the sequence associated to a valuation $\nu$ (or equivalently to a sequence $(*))$. Therefore these properties characterize the class of all valuations with the same associated sequence $\delta_{\nu}$. This gives rise to a notion of equisingularity of valuations.

For this, we see that all such sequence can be realized taking $R=\mathbb{Q}\left(t_{1}, \ldots, t_{n}, \ldots\right)[[X, Y]], \mathbb{Q}$ being the field of rational numbers. In general, this is not possible for any $R$. If, in addition, the sequence satisfies that $\delta(j)$ is an integer for all $j \geq 0$ (or equivalently all rings of $(*)$ have the same residue field) then there is a valuation $\nu$ such that its associated proximity sequence is the given one.

We are also interested in other properties of the proximity sequence. In particular, if $R$ is a complete ring then there is a non-zero 
principal prime ideal $J$ of $R$ such that $J$ "goes through" $R_{n}$ for all $n \geq 0$ (i.e. $J_{n} \neq R_{n}$, where $J_{n}$ is the strict quadratic transform of $J$ in $\left.R_{n}\right)$ if and only if there is $N_{0}$, such that $\delta_{\nu}(n)=0$ for all $n \geq N_{0}$. In this situation, $\delta_{\nu}$ characterizes the equisingularity classes of analytically irreducible plane curves. So we also have an explicit description of the different equisingularity classes.

The paper is organized as follows:

In Section 1 we outline some definitions and properties of proximity relations.

Section 2 is devoted to an introduction of the invariant and to study its properties. In particular we see that it is equivalent to the refined proximity matrix.

In the last section we characterize $\delta_{\nu}$ by its properties and when $\delta_{\nu}$ is an invariant for the equisingularity of plane curves.

\section{Preliminaries.}

First we will outline some concepts about the proximity relations of $(*)$.

For $j>i$ we say that $R_{j}$ is proximate to $R_{i}$ if the valuation ring $V\left(R_{i}\right)$ of $\operatorname{Ord}_{R_{i}}$ contains $R_{j}$, where $\operatorname{Ord}_{R_{i}}$ is the usual valuation order of $R_{i}$ (i.e. $\operatorname{Ord}_{R_{i}}(x)$ is the greatest non-negative integer $d$ such that $x \in\left(M\left(R_{i}\right)\right)^{d}, x$ being a non-zero element of $\left.R_{i}\right)$. In this case, $V\left(R_{i}\right)=$ $\left(R_{j}\right)_{\mathfrak{p}}$, where $\mathfrak{p}$ is a height one prime ideal of $R_{j}$ containing $M\left(R_{i}\right) R_{j}$ and $R_{k}$ is proximate to $R_{i}$ for $i<k \leq j$.

Moreover, for $j>i$ it is easy to verify that $M\left(R_{i}\right) R_{j}=t_{i j}^{a_{i j}} u_{i j}^{b_{i j}}$, where $t_{i j} R_{j}=M\left(R_{j-1}\right) R_{j},\left(t_{i j}, u_{i j}\right) R_{j}=M\left(R_{j}\right), a_{i j}>0$ and $b_{i j} \geq 0$. ( $a_{i j}$ and $b_{i j}$ being integers). So $R_{j}$ is proximate to $R_{j-1}$ and at most to one other ring in $(*)$. In fact if $j>i+1$ and $R_{j}$ is proximate to $R_{i}$ we can write

$$
R_{k}=\left(R_{k-1}\left[\frac{u_{i, k-1}}{t_{i, k-1}}\right]\right)_{\left(t_{i, k}, u_{i, k}\right)}
$$

with $t_{i, k}=t_{i, k-1}$ and $u_{i, k}=u_{i, k-1} / t_{i, k-1}, i+2 \geq k \geq j$. So $b_{i, j}=1$ and this is also a sufficient condition for $R_{j}$ to be proximate to $R_{i}$.

One also has $a_{i, j}=j+i-1$ and $e_{k-1}=1, i+2 \leq k \leq j$.

In general, for $j>i+1$ we say that $R_{j}$ is a satellite of $R_{i}$ if $b_{i j} \neq 0$, where $M\left(R_{i}\right) R_{j}=t_{i j}^{a_{i j}} u_{i j}^{b_{i j}}$ as above. If $b_{i j}=0$ we say that $R_{j}$ is free with respect to $R_{i}$. 
This is simply Zariski's definition of satellite and free points. (See [17]). It should be noted that $R_{j}$ is a satellite of $R_{i}$ if and only if $\operatorname{Ord}_{R_{j}}\left(\sqrt{M\left(R_{i}\right) R_{j}}\right)=2$. It is also easy to verify that $R_{j}$ is a satellite of $R_{i}$ if and only if there is a non-negative integer $q$ with $j-1>q \geq i$ such that $R_{j}$ is proximate to $R_{q}$.

\section{The invariant.}

In this section we will use the above notations.

We define the function $\gamma: \mathbb{Z}_{0} \longrightarrow \mathbb{Z}_{0}$ as follows: $\gamma(0)=0$ and for $j \geq 1, \gamma(j)=1+\min \left\{k: R_{j}\right.$ is proximate to $\left.R_{k}\right\}$, where $\mathbb{Z}_{0}$ denotes the set of non-negative integers.

Thinking geometrically, this map computes the oldest exceptional divisor that "goes through" $R_{j}$.

On the other hand, note that $\gamma(j)<j$ if and only if $R_{j}$ is a satellite of $R_{i}$ for some $i<j-1$. So $\gamma(j)=j$ if and only if $R_{j}$ is free with respect to $R_{i}$ for all $i<j-1$.

The most interesting properties of $\gamma$ are given in the following results.

Proposition 2.1. We have the following statements:

a) $\gamma(j) \leq j$

b) If $\gamma(j)<i<j$ then $\gamma(i)=\gamma(j)$.

c) For all $j \geq 0$ there is a non-negative integer $n$ such that $\gamma^{n}(j)=$ $\gamma^{n+1}(j)$, where $\gamma^{0}=\mathbf{1}_{\mathbb{Z}_{0}}$ and $\gamma^{k+1}=\gamma \circ \gamma^{k}$.

d) If $\gamma(j)<j$ then $\gamma(j)=j-1$ or $\gamma(j)=\gamma(j-1)$.

Proof. a) Follows from the definition of $\gamma$.

b) If $m+1=\gamma(j)<j$ then $R_{j}$ is proximate to $R_{m}$ and also $R_{i}$ is proximate to $R_{m}$ for $m+1 \leq i \leq j$. So $\gamma(i)=m+1=\gamma(j)$.

c) By a) we have $0 \leq \cdots \leq \gamma^{k}(j) \leq \cdots \leq \gamma(j) \leq j$. So there is an $n$ such that $\gamma^{n}(j)=\gamma^{n+1}(j)$.

d) As $\gamma(j)<j$, if $\gamma(j) \neq j-1$ then $\gamma(j)<j-1<j$ by a). And by b) $\gamma(j)=\gamma(j-1)$. 
In what follows we will denote by

$$
n(j)=\min \left\{n \in \mathbb{Z}_{0}: \gamma^{n}(j)=\gamma^{n+1}(j)\right\} .
$$

Proposition 2.2. With the above notations, let us assume that $k<j$, then we have:
1) If $\gamma(j)=k$ then $n(j)=n(k)+1$.
2) If $\gamma(j)=\gamma(k)$ then $n(j)=n(k)$.

ProOF. Note that $\gamma^{n(k)+1}(j)=\gamma^{n(k)}(k)=\gamma^{n(k)+1}(k)=\gamma^{n(k)+2}(j)$, so $n(j) \leq n(k)+1$.

On the other hand, $\gamma^{n(j)-1}(k)=\gamma^{n(j)}(j)=\gamma^{n(j)+1}(j)=\gamma^{n(j)}(k)$, so $n(j) \geq n(k)+1$ and we have 1$)$.

The proof of 2) is similar.

Now we have the conditions to define the invariant, which we will call proximity sequence.

We define $\delta_{\nu}=\left\{\delta_{\nu}(j)\right\}_{j \geq 0}$ as follows: $\delta_{\nu}(0)=0$ and for $j \geq 1$

$$
\delta_{\nu}(j)=n(j)+1-\frac{1}{e_{j-1}} .
$$

First of all, we will see that the sequence $\delta_{\nu}$ characterizes the proximity relations of $\nu$ (or equivalently of $(*)$ ).

Proposition 2.3. With the above notations, the following statements are equivalent:

a) $R_{j}$ is free with respect to $R_{i}$ for all $i<j-1$.

b) $\delta_{\nu}(j)=1-\left(1 / e_{j-1}\right)$.

c) $\delta_{\nu}(j)<1$.

ProOF. $R_{j}$ is free with respect to $R_{i}$ for all $i<j-1$ if and only if $\gamma(j)=j$, so if and only if $\delta_{\nu}(j)=1-\left(1 / e_{j-1}\right)$ or equivalently $\delta_{\nu}(j)<1$.

Proposition 2.4. With the above notations, if $i<j-1$ the following statements are equivalent:

a) $R_{j}$ is proximate to $R_{i}$. 
b) $n(i+1)+1=\delta_{\nu}(i+1)+1 / e_{i}=\delta_{\nu}(k)=\delta(j), i+2 \leq k \leq j$.

Proof. In order to see that a) implies b), we note that $R_{k}$ is proximate to $R_{i}, i+1 \leq k \leq j$. So $\gamma(k)=i+1$ for $i+2 \leq k \leq j$. Then, by definition of $\delta_{\nu}$ we have $\delta_{\nu}(i+1)+1 / e_{i}=1+n(i+1)=n(k)=\delta_{\nu}(k)$, $i+2 \leq k \leq j$.

On the other hand, by Proposition $2.3 R_{j}$ is proximate to $R_{h}$, $h<j-1$.

If $h<i$ then by a) implies b) we have that $\delta_{\nu}(k)=\delta_{\nu}(j)$ for $h+2 \leq k \leq j$. In particular, $\delta_{\nu}(i+1)=\delta_{\nu}(j)$. Yet $\delta_{\nu}(i+1)<$ $n(i+1)+1=\delta_{\nu}(j)$, which is a contradiction.

If $i<h$ then also by a) implies b) $\delta_{\nu}(h+1)<\delta_{\nu}(j)$, which is also a contradiction.

So $h=i$ and we have that b) implies a).

Proposition 2.5. With the above notations, the proximity sequence $\delta_{\nu}$ has the following properties:

1) $\delta_{\nu}(j) \geq 0$.

2) $\delta_{\nu}(0)=0$.

3) $\delta_{\nu}(1)<1$.

4) If $\delta_{\nu}(j) \geq 1$ then $\delta_{\nu}(i)$ is an integer.

5) If $\delta_{\nu}(j)<1$ then $1 /\left(1-\delta_{\nu}(j)\right)$ is an integer.

6) If $\delta_{\nu}(j+1)<\delta_{\nu}(j)$ then $\delta_{\nu}(j+1)<1$.

7) $\delta_{\nu}(j+1) \leq 1+\delta_{\nu}(j)$.

Proof. 1) and 2) follow from the definition of $\delta_{\nu}$.

3) As $\gamma(1)=1$ we have $n(1)=0$ and $\delta_{\nu}(1)=1-1 / e_{1}<1$.

4) If $\delta_{\nu}(j) \geq 1$ then $\gamma(j) \neq j$, so $R_{j}$ is proximate to $R_{q}$, with $q<j-1$. So $e_{j-1}=1$ and $\delta_{\nu}(j)=n(j)$ is an integer.

5) If $\delta_{\nu}(j)<1$ then $\gamma(j)=j$ and $\delta_{\nu}(j)=1-1 / e_{j-1}$, so $e_{j-1}=$ $1 /\left(1-\delta_{\nu}(j)\right)$ is an integer.

6) If $\delta_{\nu}(j+1)<\delta_{\nu}(j)$ and $\delta_{\nu}(j+1) \geq 1$, then $\delta_{\nu}(j) \geq 1$. So $R_{j+1}$ is proximate to $R_{q}, q<j$ and $R_{j}$ is proximate to $R_{h}, h<j-1$. Therefore $\gamma(j+1)=q+1, \gamma(j)=h+1, e_{j-1}=e_{j}=1, \delta_{\nu}(j+1)=n(j+1)=$ $n(q+1)+1$ and $\delta_{\nu}(j)=n(j)=n(h+1)+1$. 
If $q<j-1$ then $q=h$ and $\delta_{\nu}(j+1)=\delta_{\nu}(j)$, which is a contradiction.

So $q=j-1$ and $\gamma(j+1)=j$. Then by 2.2 we have $n(j+1)=$ $n(j)+1$ and

$$
\delta_{\nu}(j+1)=n(j+1)+1-\frac{1}{e_{j}}=n(j)+1+1-\frac{1}{e_{j-1}}=\delta_{\nu}(j)+1,
$$

which is also a contradiction. So $\delta_{\nu}(j+1)<1$.

7) We have three possibilities:

- $\gamma(j+1)=j+1$, in this case $\delta_{\nu}(j+1)<1$ and always $\delta_{\nu}(j+1) \leq$ $\delta_{\nu}(j)+1$.

- $\gamma(j+1)=j$, in this case we have $n(j+1)=n(j)+1$, see 2.2. So

$$
\delta_{\nu}(j+1)=n(j+1)+1-\frac{1}{e_{j}} \leq \delta_{\nu}(j)+1
$$

- $\gamma(j+1)=\gamma(j)$, in this case we have $n(j+1)=n(j)$, see 2.2. So

$$
\delta_{\nu}(j+1)=n(j+1)+1-\frac{1}{e_{j}}
$$

and

$$
\delta_{\nu}(j)=n(j)+1-\frac{1}{e_{j-1}}
$$

then

$$
\delta_{\nu}(j+1)=\delta_{\nu}(j)+\frac{1}{e_{j-1}}-\frac{1}{e_{j}}<\delta_{\nu}(j)+1
$$

To finish this section we will compare the proximity sequence with other invariants. Namely, we will see that it defines equivalent data to the refined proximity matrix.

It should be remembered (see [13]) that the refined proximity ma$\operatorname{trix} P_{\nu}=\left(p_{i j}\right)_{i, j \geq 0}$ is given by $p_{i i}=1$,

$$
p_{i j}=-\left[\frac{R_{j}}{M\left(R_{j}\right)}: \frac{R_{i}}{M\left(R_{i}\right)}\right]
$$

if $R_{j}$ is proximate to $R_{i}$ and $p_{i j}=0$ for the rest. Note that $P_{\nu}$ is an upper triangular matrix. 
Proposition 2.6. The proximity sequence $\delta_{\nu}$ determines the refined proximity matrix $P_{\nu}$ and vice-versa.

Proof. First we note that $p_{00}=1, p_{10}=0$ and

$$
p_{01}=-\left[\frac{R_{1}}{M\left(R_{1}\right)}: \frac{R_{0}}{M\left(R_{0}\right)}\right]=-e_{0}=\frac{1}{\delta_{\nu}(1)-1} .
$$

So $p_{01}$ and $\delta_{\nu}(1)$ are the same data.

Now let us assume that $\delta_{\nu}$ determines $p_{i j}$ for $0 \leq i, j \leq n, n \geq 1$. We have $p_{n+1, n+1}=1$ and $p_{n+1, k}=0$ for $0 \leq k \leq n$.

If $R_{n+1}$ is free with respect to $R_{k}$ for all $k<n$, then $p_{k, n+1}=0$ for $k<n$ and

$$
p_{n, n+1}=-\left[\frac{R_{n+1}}{M\left(R_{n+1}\right)}: \frac{R_{n}}{M\left(R_{n}\right)}\right]=-e_{n}=\frac{1}{\delta_{\nu}(n+1)-1} .
$$

If $R_{n+1}$ is proximate to $R_{k}$ with $k<n$ then

$$
n(k+1)+1=\delta_{\nu}(k+2)=\delta_{\nu}(k+3)=\cdots=\delta_{\nu}(n+1)=\delta_{\nu}(k)+\frac{1}{e_{k}} .
$$

So

$$
\frac{1}{\delta_{\nu}(n+1)-\delta_{\nu}(k+1)}=p_{k, n+1}
$$

Now

$$
p_{n, n+1}=-\left[\frac{R_{n+1}}{M\left(R_{n+1}\right)}: \frac{R_{n}}{M\left(R_{n}\right)}\right]=-e_{n}=-1
$$

and $p_{j, n+1}=0$ for $j<n$, and $j \neq k$.

So $\delta_{\nu}$ determines $P_{\nu}$.

Similar reasoning proves that $P_{\nu}$ determines $\delta_{\nu}$.

\section{Valuations with a given $\delta$.}

Now we will prove the main result of this paper.

Theorem 3.1. Let $\delta=\{\delta(j)\}_{j \geq 0}$ be a sequence of non-negative rational numbers having the seven properties of Proposition 2.5. Then there is a two dimensional regular noetherian local ring $R$ and a valuation $\nu$ centered at $M(R)$ such that its proximity sequence is $\delta$. 
Proof. We consider $R=\mathbb{Q}\left(t_{1}, \ldots, t_{n}, \ldots\right)[[X, Y]]$, where $\mathbb{Q}$ is the field of rational numbers, $\left\{t_{1}, \ldots, t_{n}, \ldots\right\}$ is a set of indeterminates over $\mathbb{Q}$ and $X$ and $Y$ are two indeterminates over $\mathbb{Q}\left(t_{1}, \ldots, t_{n}, \ldots\right)$.

We define $e_{j-1}=1$ if $\delta(j) \geq 1$ and

$$
e_{j-1}=\frac{1}{1-\delta(j)}, \quad \text { if } \delta(j)<1 .
$$

We put $R=R_{0}$ and

$$
R_{1}=\left(R\left[\frac{Y}{X}\right]\right)_{\left(X,(Y / X)^{e_{0}}-t_{1}\right)} .
$$

Now let us assume that for $n \geq 1$ we have $R=R_{0} \subset R_{1} \subset \cdots \subset R_{n}$ such that for any valuation $\nu^{\prime}$ centered at $M\left(R_{n}\right)$ we have that $\delta_{\nu^{\prime}}(j)=\delta(j)$, for each $0 \leq j \leq n$, and

$$
\frac{R_{j}}{M\left(R_{j}\right)}=\frac{R_{j-1}}{M\left(R_{j-1}\right)}\left[t_{j}^{1 / e_{j-1}}\right], \quad \text { if } e_{j-1}>1
$$

and

$$
\frac{R_{j}}{M\left(R_{j}\right)}=\frac{R_{j-1}}{M\left(R_{j-1}\right)}, \quad \text { if } e_{j-1}=1,1 \leq j \leq n .
$$

We have two possibilities:

1) $\delta(n+1)<1$ (i.e. $R_{n+1}$ must be free with respect to $R_{i}$ for all $i<n)$. In this case, let $\left(x_{n}, y_{n}\right)$ be a basis of $M\left(R_{n}\right)$, such that $M\left(R_{n-1}\right) R_{n}=x_{n} R_{n}$.

We define

$$
R_{n+1}=\left(R_{n}\left[\frac{y_{n}}{x_{n}}\right]\right)_{\left(x_{n},\left(y_{n} / x_{n}\right)^{e_{n}}-t_{n+1}\right)} .
$$

2) $\delta(n+1) \geq 1$ (i.e. $R_{n+1}$ must be a satellite). In this case, we have $1+\delta(n) \geq \delta(n+1) \geq \delta(n)$.

- If $\delta(n+1)>\delta(n)$, then $R_{n+1}$ must be proximate to $R_{n-1}$. (See $2.4)$. Let $\left(x_{n}, y_{n}\right)$ be a basis of $M\left(R_{n}\right)$, such that $M\left(R_{n-1}\right) R_{n}=x_{n} R_{n}$.

We define

$$
R_{n+1}=\left(R_{n}\left[\frac{x_{n}}{y_{n}}\right]\right)_{\left(y_{n}, x_{n} / y_{n}\right)} .
$$

- If $\delta(n+1)=\delta(n)$, then $R_{n+1}$ must be proximate to $R_{k}$, with $k<n-1$. (See 2.4). In this case, we can take $\left(x_{n}, y_{n}\right)$ a basis of $M\left(R_{n}\right)$, such that $M\left(R_{n-1}\right) R_{n}=x_{n} R_{n}$ and $M\left(R_{k}\right) R_{n}=x_{n}^{a} y_{n} R_{n}$. 
We define

$$
R_{n+1}=\left(R_{n}\left[\frac{y_{n}}{x_{n}}\right]\right)_{\left(x_{n}, y_{n} / x_{n}\right)} .
$$

Now it is easy to see that $R=R_{0} \subset R_{1} \subset \cdots \subset R_{n} \subset R_{n+1}$ proves that for any valuation $\nu^{\prime}$ centered at $M\left(R_{n+1}\right)$ we have $\delta_{\nu^{\prime}}(j)=\delta(j)$, for each $0 \leq j \leq n+1$, and

$$
\frac{R_{j}}{M\left(R_{j}\right)}=\frac{R_{j-1}}{M\left(R_{j-1}\right)}\left[t_{j}^{1 / e_{j-1}}\right], \quad \text { if } e_{j-1}>1
$$

and

$$
\frac{R_{j}}{M\left(R_{j}\right)}=\frac{R_{j-1}}{M\left(R_{j-1}\right)}, \quad \text { if } e_{j-1}=1,1 \leq j \leq n+1
$$

Now we will study the case in which $\delta_{\nu}(j)$ is an integer for all $j \geq 0$.

Theorem 3.2. Let $\delta=\{\delta(j)\}_{j \geq 0}$ be a sequence of non-negative integers having the seven properties of Proposition 2.5. Let $R$ be any regular noetherian local ring of dimension two. Then there is a valuation $\nu$ centered at $M(R)$ such that its proximity sequence is $\delta$.

Proof. First we put $e_{j-1}=1$ for all $j \geq 0, R=R_{0}$ and

$$
R_{1}=\left(R\left[\frac{y}{x}\right]\right)_{(x, y / x)}
$$

$(x, y)$ being any basis of $M(R)$.

Now let us assume that we have $R=R_{0} \subset R_{1} \subset \cdots \subset R_{n}$ such that for any valuation $\nu^{\prime}$ centered at $M\left(R_{n}\right)$ we have $\delta_{\nu^{\prime}}(j)=\delta(j)$, for each $0 \leq j \leq n$,

$$
\frac{R_{j}}{M\left(R_{j}\right)}=\frac{R_{j-1}}{M\left(R_{j-1}\right)}, \quad 1 \leq j \leq n
$$

We have two possibilities:

1) $\delta(n+1)=0$ (i.e. $R_{n+1}$ must be free with respect to $R_{i}, i<n$ ). In this case, let $\left(x_{n}, y_{n}\right)$ be a basis of $M\left(R_{n}\right)$, such that $M\left(R_{n-1}\right) R_{n}=$ $x_{n} R_{n}$.

We define

$$
R_{n+1}=\left(R_{n}\left[\frac{y_{n}}{x_{n}}\right]\right)_{\left(x_{n},\left(y_{n} / x_{n}\right)\right)} .
$$


2) $\delta(n+1) \geq 1$ (i.e. $R_{n+1}$ must be a satellite). In this case, we have $1+\delta(n) \geq \delta(n+1) \geq \delta(n)$.

- If $\delta(n+1)>\delta(n)$, then $R_{n+1}$ must be proximate to $R_{n-1}$. (See $2.4)$. Let $\left(x_{n}, y_{n}\right)$ be a basis of $M\left(R_{n}\right)$, such that $M\left(R_{n-1}\right) R_{n}=x_{n} R_{n}$.

We define

$$
R_{n+1}=\left(R_{n}\left[\frac{x_{n}}{y_{n}}\right]\right)_{\left(y_{n}, x_{n} / y_{n}\right)} .
$$

- If $\delta(n+1)=\delta(n)$, then $R_{n+1}$ must be proximate to $R_{k}$, with $k<n-1$. (See 2.4). In this case, we can take $\left(x_{n}, y_{n}\right)$ a basis of $M\left(R_{n}\right)$, such that $M\left(R_{n-1}\right) R_{n}=x_{n} R_{n}$ and $M\left(R_{k}\right) R_{n}=x_{n}^{a} y_{n} R_{n}$.

We define

$$
R_{n+1}=\left(R_{n}\left[\frac{y_{n}}{x_{n}}\right]\right)_{\left(x_{n}, y_{n} / x_{n}\right)} .
$$

Now it is easy to see that $R=R_{0} \subset R_{1} \subset \cdots \subset R_{n} \subset R_{n+1}$ proves that for any valuation $\nu^{\prime}$ centered at $M\left(R_{n+1}\right)$ we have $\delta_{\nu^{\prime}}(j)=\delta(j)$, for each $0 \leq j \leq n+1$, and

$$
\frac{R_{j}}{M\left(R_{j}\right)}=\frac{R_{j-1}}{M\left(R_{j-1}\right)}, \quad 1 \leq j \leq n+1 .
$$

It should be noted that the above theorem is not true if $\delta$ is not a sequence of non-negative integers.

For example, let us consider $R=\mathbb{R}[[X, Y]]$, where $\mathbb{R}$ is the field of real numbers. Let $\delta=\{\delta(j)\}_{j \geq 0}$ be the sequence given by $\delta(0)=0$, $\delta(1)=2 / 3$ and $\delta(k)=0$ for $k \geq 2$. If there is a valuation $\nu$ (or equivalently a sequence $(*))$ with $\delta$ as the proximity sequence, then $R / M(R)$ is isomorphic to $\mathbb{R}$ and

$$
e_{0}=\left[\frac{R_{1}}{M\left(R_{1}\right)}: \frac{R}{M(R)}\right]=3,
$$

which is a contradiction.

To finish the paper, we will clarify the relation between the proximity sequence and the classification of plane curve singularities.

For this, we need to assume that $R$ is a complete ring.

Proposition 3.3. Let us assume that there is a non-zero principal prime ideal $J$ of $R=R_{0}$ such that $J_{n} \neq R_{n}, J_{n}$ being the strict quadratic transform of $J$ in $R_{n}, n \geq 0$. Then there is a non-negative integer $N_{0}$ such that $\delta_{\nu}(n)=0$ for $n \geq N_{0}$. 
Proof. By [2, Proposition 9.4 and Theorem 10.7], there is an $N_{0}$ such that $J R_{n}$ has a normal crossing for $n \geq N_{0}$, that is $J R_{n}=x_{n}^{a_{n}} y_{n}^{b_{n}} R_{n}$, where $\left(x_{n}, y_{n}\right)$ is a basis of $R_{n}$ and $a_{n}$ and $b_{n}$ are non-negative integers.

On the other hand, by definition of strict quadratic transform of $J$ we have

$$
J R_{n}=\left(\prod_{i=0}^{n-1}\left(M\left(R_{i}\right)\right)^{d_{i}}\right) J_{n} R_{n},
$$

where $d_{i}=\operatorname{Ord}_{R_{i}}\left(J_{i}\right), 0 \leq i \leq n-1$.

We can thus assume that $J_{n}=y_{n} R_{n}$, with $b_{n}=1$ and

$$
\prod_{i=0}^{n-1}\left(M\left(R_{i}\right)\right)^{d_{i}} R_{n}=x_{n}^{a_{n}} R_{n}
$$

Therefore $R_{n}$ is free for $n \geq N_{0}$.

As $J_{n+1} \neq R_{n+1}$ we have

$$
R_{n+1}=\left(R_{n}\left[\frac{y_{n}}{x_{n}}\right]\right)_{\left(x_{n}, y_{n} / x_{n}\right)}
$$

so $e_{n}=1$, for $n \geq N_{0}$.

Now, we have $\gamma(n)=n$ and $\delta(n)=0$ for $n \geq N_{0}$.

Proposition 3.4. With the above notations, let us assume that there is a non-negative integer $N_{0}$ such that $\delta_{\nu}(n)=0$ for $n \geq N_{0}$. Then, there is a non-zero principal prime ideal $J$ of $R=R_{0}$ such that $J_{n} \neq R_{n}, J_{n}$ being the strict quadratic transform of $J$ in $R_{n}$ for all $n \geq 0$.

Proof. As $\delta_{\nu}(n)=0$ for $n \geq N_{0}$ we have that $R_{n}$ is free and $e_{n}=1$, for $n \geq N_{0}$.

So we can write

$$
R_{n+1}=\left(R_{n}\left[\frac{y_{n}}{x_{n}}\right]\right)_{\left(x_{n},\left(y_{n} / x_{n}\right)+a\right)},
$$

where $\left(x_{n}, y_{n}\right)$ is a basis of $M\left(R_{n}\right)$ and $a_{n} \in R_{N_{0}}, n \geq N_{0}$.

Let us consider the ideal

$$
J_{N_{0}}=\left(y_{N_{0}}+a_{N_{0}} x_{N_{0}}+a_{N_{0}+1} x_{N_{0}}^{2}+\cdots\right)\left(R_{N_{0}}\right)^{*}
$$

where $\left(R_{N_{0}}\right)^{*}$ is the complection of $R_{N_{0}}$. 
It is now easy to see that $J=J_{N_{0}} \cap R$ is the required non-zero principal prime ideal of $R$.

It should be noted that Propositions 3.3 and 3.4 characterize the proximity sequences such that there is an analytically irreducible plane curve that "goes through" all the rings of $(*)$.

In addition, it is easy to verify that $\delta_{\nu}$ is an invariant of the equisingularity class of such a curve. For a more specific treatment of proximity relations and plane curve singularities refer to [12].

\section{References.}

[1] Abhyankar, S. S., On the valuations centered in a local domain. Amer. J. Math. 78 (1956), 321-348.

[2] Abhyankar, S. S., Desingularization of plane curves. In Proceedings Symposia Pure Mathematics, Part 1. 40 (1983), 1-45.

[3] Aparicio, J., Clasificación de Singularidades de Curvas Planas y Valoraciones Divisoriales. Ph. D. Thesis, University of Valladolid, 1992.

[4] Bourbaki, N., Algebre Commutative. Chapitres 8 et 9. Masson, 1983.

[5] Bourbaki, N., Algebre Commutative. Chapitres 5 a 7. Masson, 1985.

[6] Enriques, F., Chisini, O., Lezioni sulla teoria geometrica delle equazioni e delle funcioni algebriche. Vol. II, N. Zanichelli, 1918.

[7] Campillo, A., Gonzalez-Sprinberg, G., Lejeune-Jalabert, M., Amas, ideaux a support fini et chaines toriques. C.R. Acad. Sci. Paris Serie I. 315 (1992), 987-990.

[8] Campillo, A., Gonzalez-Sprinberg, G., Lejeune-Jalabert, M., Clusters of infinitelly near points. Math. Ann. 306 (1996), 169-194.

[9] Campillo, A., Reguera, A. J., Combinatorial aspects of sequences of point blowing ups. Manuscripta Math. 84 (1994), 29-46.

[10] Granja, A., Coefficient fields for plane curves and equisingularity. Comm. Algebra 18 (1990), 193-208.

[11] Granja, A., Equisingularity invariants of plane curves. In: Algebraic Geometry and its Applications. Springer-Verlag, 1994, 89-12.

[12] Granja, A., Sánchez-Giralda, T., Enriques graphs of plane curves. Comm. Algebra 20 (1992), 527-562.

[13] Lipman, J., Proximity inequalities for complete ideals in two-dimensional regular local rings. Contemporary Math. 159 (1994), 293-306.

[14] Northcott, D. G., Abstract dilatations and infinitely near points. Proc. Cambridge Phil. Soc. 52 (1956), 176-197. 
[15] Spivakovsky, M., Valuations in function fields of surfaces. Amer. J. Math. 112 (1990), 107-156.

[16] Zariski, O., Studies in equisingularity I. Amer. J. Math. 87 (1965), 507-536.

[17] Zariski, O., General Theory of Saturation and of Saturated Local Rings II. Amer. J. Math. 93 (1971), 872-964.

[18] Zariski, O., Samuel, P., Commutative Algebra. Vol. I and II. SpringerVerlag, 1986.

Recibido: 25 de junio de 1.998

José Juan Aparicio

Departamento de Algebra, Geometría y Topología Universidad de Valladolid 47005 Valladolid, SPAIN aparicio@cpd.uva.es

Angel Granja* Departamento de Matemáticas Universidad de León 24071 León, SPAIN demagb@isidoro.unileon.es and

Tomás Sánchez-Giralda Departamento de Algebra, Geometría y Topología Universidad de Valladolid 47005 Valladolid, SPAIN sanchezgiral@cpd.uva.es

\footnotetext{
* Partially supported by DGICYT PB95-0603-C02 and Le09/95
} 